\title{
Using Fuzzy Evaluation Decision Model to Assess the Operation Scheme of Taxi in Shanghai Pudong Airport
}

\author{
Botao Liu*, Boying Lv, Yishuai Tian ${ }^{1}$
}

${ }^{1}$ College of Hydraulic \& Environmental Engineering, China Three Gorges University, Yichang 443002, China.

\section{ABSTRACT}

In order to investigate the decision of the airport taxi driver whether to return to carry passengers, we established a fuzzy

*Correspondence to Author: evaluation decision model based on the analytic hierarchy Botao Liu

process, combined with the application analysis of Shanghai College of Hydraulic \& EnvironmenPudong Airport. Based on the selection and quantification of decision indicators, we used the AHP method to calculate the China. weights to eliminate indicators and optimize the indicator system. Then, a two-level fuzzy evaluation model was established, and the Bayesian discriminant verification model was more reasonable. Finally, based on the data analysis of Shanghai Pudong Airport, combined with 16 different airport decisiontal Engineering, China Three Gorgmaking situations, the model was used to obtain the no-load return trips and the membership of waiting passengers. We compared and selected the larger membership value as the decision. Besides, the dependence of the model was analyzed, and it was found that the main dependent factors for decision- 2:8 making were the number of flight arrivals and the driver's arrival time. After solving the above, we provide relevant basis for the decision of airport taxi drivers.

How to cite this article:

Botao Liu, Boying Lv, Yishuai Tian. Using Fuzzy Evaluation Decision Model to Assess the Operation Scheme of Taxi in Shanghai Pudong Airport. International Journal of Trade and Policy, 2020;

Keywords: Fuzzy evaluation; AHP; Airport taxi; Bayesian discriminant

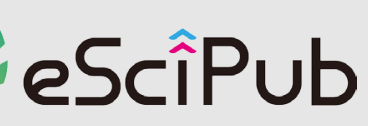

eSciPub LLC, Houston, TX USA. Website: http://escipub.com/ 


\section{Introduction}

When taxi drivers arrive at an airport or a largescale transportation hub, they will decide whether to stay in the storage pool or wait for passengers, and whether to stay will cause changes in the driver's income. At this time, the driver needs to take into account the comprehensive determinate and uncertain factors ${ }^{[1]}$. If the waiting time is too long, it will cause time cost loss. If the driver chooses to return to the urban area empty, it will lose the potential passenger income and pay additional empty charge. In addition to the time cost of waiting in line, which will affect the decision-making of taxi drivers, it may also be related to factors such as the size of the airport city, the peak flow of people, rainy and snowy weather, the arrival density of flights in a certain period of time, etc. ${ }^{[2-4]}$, with different correlations and different effects. Therefore, it is of great significance to study the decision-making strategies of taxi drivers' influencing factors.

Taxi as one of important part of urban transportation system, the current decision road for taxi drivers have a lot of research, for example: Loukas Dimitriou et al. ${ }^{[3]}$ for large-scale data set from New York City were analyzed, visualization and rendering, so as to provide evidence that showed the advanced dynamic mapping tool deployment in estimating the taxi operation best strategy advantage. Bao Feng ${ }^{[5]}$ taking hongqiao comprehensive transportation hub as an example, introduced the determination method of the scale of taxi arrival and departure passenger points in the supporting facilities of hub transportation. Yong Han et al. ${ }^{[6]}$ optimized the parameters of the traditional DBSCAN clustering algorithm and the taxi passenger point data clustering analysis, analysis of the research area of the taxi passenger point distribution, different dates, taxi passenger area in the different periods of time and space distribution of change, reflected the laws of the city dweller, looking for customers to provide the reference for the taxi driver. According to the characteristics of airport taxi passenger flow data, Sirui Lin ${ }^{[7]}$ constructed an appropriate model, designed an appropriate data mining algorithm, and predicted the taxi capacity demand based on the real-time information in the current database, so as to reasonably dispatch and allocate airport taxi resources. Bingjie Wang et al. ${ }^{[8]}$ used the analytic hierarchy process to conduct qualitative and quantitative analysis on the relevant factors affecting the driver's decision-making, and established the optimal decision-making model for taxi drivers to maximize their benefits. Jing et al. ${ }^{[9]}$ constructed a mobility pattern classification model suitable for taxi OD (original destination) point data, and used DBSCAN algorithm to classify time series into different mobility patterns based on the proximity index calculated using the improved similarity measure. Xu et al. ${ }^{[10]}$ studied the researcher flow model, focusing on the travel modes of taxi passengers by examining the data sets of three major cities. Through statistical analysis, it is found that the lognormal distribution with power law tail can best approximate the displacement and duration of taxi trip in the investigated cities. Maguire et al. ${ }^{[11]}$ analyzed the changes of drivers' psychological pressure on driving intention. However, most of the above studies were path decision analysis of taxi drivers. However, there were relatively few quantitative studies on whether taxi drivers choose to stay or not to take passengers and seek to maximize drivers' benefits. Some of them were only analyzed through spatial and temporal OD ${ }^{[12]}$, without clearly discussing the degree of influencing factors.

Therefore, based on the existing taxi GPS data analysis method [13], this paper has taken Pudong Airport in Shanghai, China as an example to collect flight data and GPS data of 10,000 taxis in the urban area of Shanghai. This paper analyzed the decision-making factors and influencing mechanism of taxi drivers, established a decision model based on AHP, and designed some basic situation simulation and gave the decision scheme, thus providing relevant basis for the study of taxi drivers' decision to stay.

\section{Articulate our metrics}

\subsection{Selection and quantification of indicators}

Before establishing an airport taxi decision-making model, this article does not consider the assumption of the influence of psychological 
factors on the driver. The driver's emotions will cause changes in the decision-making [14]. Therefore, the influencing factors of taxi decision-making are first selected as the indicators of the decision-making model, and the quantitative indicators are conveniently substituted into the calculation. The following is the selection and

$$
W_{3}=\frac{O_{i}}{\sum_{i=1}^{24} O_{i}} \quad(i=1,2, \cdots, 24)
$$

The impact of online car hire. With the increase in the number of online taxis such as "Didi Taxi" and "Uber", there is increasing competition pressure for airport taxis to return to urban areas to solicit passengers. Assume that for the same city, the impact of network rides is always the same. Suppose the impact of online car hire is $W_{1}$, and $W_{1} \in[0,1]$ measures the impact from large to small.

$$
W_{2}=\frac{C_{i}}{\sum_{i=1}^{24} C_{i}} \quad(i=1,2, \cdots, 24)
$$

Consideration of weather factors. Weather data is one of the important influencing factors on the airport's passenger flow. The weather in spring and autumn is suitable for flight, and the severe weather in summer and winter may cancel some flights and delay. As the weather gets worse, the need for passengers to take a taxi increases $^{[2]}$, Set up ${ }^{W_{5} \in[0,1]}$ for this, to indicate the severity of the weather. And represent (0.2(good),0.4(normal),0.6(worse),0.8(very poor)

Consideration of time factor. ${ }^{[1]}$ Every evening (after 22.00), buses and other traffic are closed. At this time, the willingness of passengers to choose a taxi increases. Checked the daily flight number from Pudong Airport, Beijing International Airport, Wuhan Tianhe Airport, etc., and found that the flight has morning and evening peaks. Daily 8:00 to $23: 00$ is the peak time of flight departure, 11:00-23:00 is the peak period of flight arrival. So choose morning and evening time effects.

Comprehensive city facts. Drivers also need to take into account the factors they will face during
The impact of the number of taxis in the storage pool. The ratio of the number of taxis arriving to the total number of taxis on the day of the driver's decision at the airport describes the degree of influence of the number of vehicles in the pool[15], let $C_{i}$ denote the number of vehicles in the airport pool at the driver's $i$-th period:

the cruise of the city, such as loss of fuel costs when they go back without load, congestion in the city, or whether there are major events (major events, traffic control). Therefore, we selected: crude oil prices, major events or events, and congestion in the city as the influencing factors.

The impact of congestion in the city. Measured by the traffic congestion coefficient [6], define o to describe the impact of urban traffic, and measure the degree of influence of urban congestion coefficient on decision-making.

\subsection{R-type Clustering analysis of influencing} factors

The R-type clustering method can study the similar relationship between variables, and aggregate each variable into several categories according to the relationship between the variables, so that the main factors affecting the system can be easily found [16]. The similarity measure adopted in this paper is the correlation coefficient, and the specific method is as follows: Let the value of the variable $x_{j}$ be 
$\left(x_{1 j}, x_{1 j}, \cdots, x_{219 j}\right)^{T} \in R^{n}(j=1,2, \cdots, 11)$. Then we two variables $x_{j}$ and $x_{k}$ as their similarity meascan use the sample correlation coefficients of the ure as:

$$
r_{j k}=\frac{\sum_{i=1}^{n}\left(x_{i j}-\bar{x}_{j}\right)\left(x_{i k}-\bar{x}_{k}\right)}{\left[\sum_{i=1}^{n}\left(x_{i j}-\bar{x}_{j}\right)^{2} \sum_{i=1}^{n}\left(x_{i k}-\bar{x}_{k}\right)^{2}\right]^{\frac{1}{2}}}
$$

The variable clustering method is used to classify the above influencing factors. The specific process is as follows:

In the longest distance method, two types of distances are defined as $R\left(G_{1}, G_{2}\right)=\max _{x_{j} \in G_{1}, x_{k} \in G_{2}}\left\{d_{j k}\right\}$

Among them: $d_{j k}=1-\left|r_{j k}\right|$ or $d^{2}{ }_{j k}=1-r_{j k}^{2}$. At this time, $R\left(G_{1}, G_{2}\right)$ is related to the similarity measure between the two variables with the smallest similarity. Finally, the correlation coefficient between the factors is used as the output parameter to obtain the decision influencing factors under three types of decision indicators.

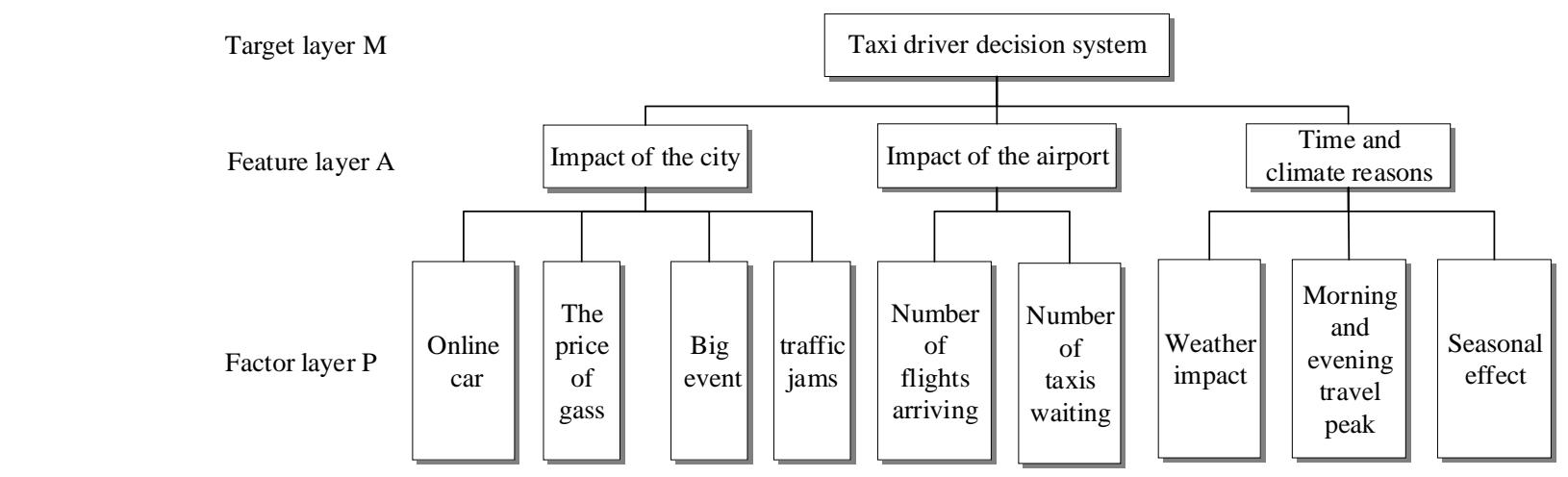

Fig 1 Hierarchy of taxi driver decision system

\section{The decision index system based on AHP 3.1 AHP system construction}

The decision problem is broken down into three levels. The uppermost layer is the target layer $\mathrm{M}$, that is, the decision-making layer that chooses to leave the airport or continue to wait [16]; the lowest layer is the factor layer, that is, the nine influencing factors P1 to P9; the middle layer is the quasi-side layer including the comprehensive urban impact $A 1$ and the airport comprehensive Three factors affect A2, time and climate, A3, as shown in Fig.1.
The first-level factor weight set is defined as $A=\left(a_{1}, a_{2}, a_{3}\right)$, and the second-level indicator's sub-weight set is $A_{1}=\left(a_{11}, a_{12}, a_{13}, a_{14}\right)$, $A_{2}=\left(a_{21}, a_{22}, a_{23}\right) \quad$ and $\quad A_{3}=\left(a_{31}, a_{32}, a_{33}\right)$.

Target layer: $\left[\begin{array}{ccc}1 & 1 / 3 & 1 / 2 \\ 3 & 1 & 3 / 2 \\ 2 & 2 / 3 & 1\end{array}\right]$

impact of the airport: $\left[\begin{array}{cc}1 & 3 / 2 \\ 2 / 3 & 1\end{array}\right]$

Temporal Climate Impact: $\left[\begin{array}{ccc}1 & 1 / 2 & 1 / 3 \\ 2 & 1 & 2 / 3 \\ 3 & 3 / 2 & 1\end{array}\right]$

First, calculate the cubic root $\bar{W}_{i}$ obtained by the product $M_{i}$ of each row of the judgment matrix
According to the data in ${ }^{[1]}$ combined with common sense, a first-level judgment matrix is constructed.
City influence:

$$
\left[\begin{array}{cccc}
1 & 1 / 2 & 1 & 1 / 2 \\
2 & 1 & 2 & 1 \\
1 & 1 / 2 & 1 & 1 / 2 \\
2 & 1 & 2 & 1
\end{array}\right]
$$


$P$, and let the vector $\bar{W}_{i}=\left(\bar{W}_{1}, \bar{W}_{2}, \bar{W}_{3}\right)$ be the normalized data obtained from the data analysis to obtain the feature vector $W=\left(W_{1}, W_{2}, W_{3}\right)$.
According to the above steps of calculating the weights, the indicators of the three types of factors are calculated respectively, and the secondlevel weight sets are obtained as follows:

$$
A_{i}=\left(a_{i j}\right)_{n} \quad(i=1,2,3, \forall j, n=1,2,3)
$$

Therefore, MATLAB software is used to program to quantify the impact of each decision index on decision factor is represented in a histogram to the taxi driver's decision. The weight of each index.

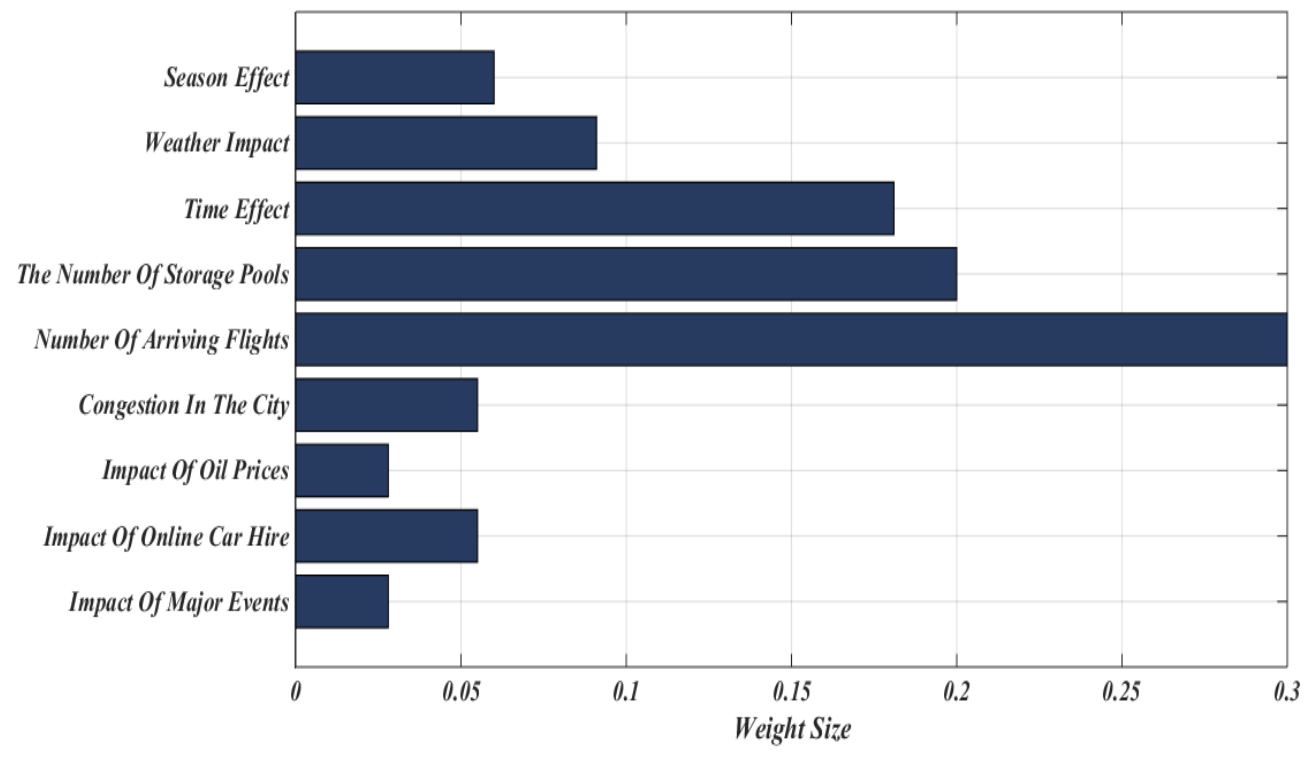

Fig 2 Histogram of the weight of each indicator

According to the analysis of weights and the actual situation of taxi driver decision-making, only the main influencing factors can be considered to simplify the influence system in order to make a decision. It can be known from the calculation results of the weights that two factors with smaller weights, such as the impact of major activities and the impact of oil prices, can be eliminated. At this point, an optimized indicator system for taxi drivers' decision impact is obtained, as shown in Table 1.

Table 1 Optimized decision impact indicator system

\begin{tabular}{c|c}
\hline \multirow{2}{*}{ Impact of the city } & $\begin{array}{c}\text { Online car } \\
\text { traffic congestion }\end{array}$ \\
\hline Impact of the airport & Number of flights arriving \\
Number of taxis waiting
\end{tabular}

4. Decision model based on fuzzy evaluation Establish an evaluation set (target layer) $V=\left(v_{1}, v_{2}\right)$, determine the evaluation set according to different goals to determine the ownership of the sample, and combine this driver selection, the evaluation set is as follows: 
$\mathrm{V}=$ (Leave the airport, Waiting in line)

Determine the participating elements (element level) $A=\left(A_{1}, A_{2}, A_{3}\right)$ The main factors affecting the taxi driver's decision are as follows:

$A=($ Comprehensive urban impact, Comprehensive impact of the airport, Temporal Climate Impact)

Establish the participating factor set (factor set) $P\left(A_{i}\right)=\left(P_{1}, P_{2}, \cdots, P_{n}\right)$. According to different goals, different influence factors are selected to establish the factor set $P\left(A_{i}\right)=\left(P_{1}, P_{2}, \cdots, P_{n}\right)$, which is the decision index. Based on the results after excluding indicators, the participating

$$
F_{1}=\left\{\begin{array}{l}
1 \quad P_{i} \geq P_{M A X} \\
\frac{P_{i}}{P_{M A X}} \quad P_{M I N} \leq P_{i} \leq P_{M A X} \\
0 \quad P_{M I N} \geq P
\end{array}\right.
$$

The membership function of the positive and negative indicators for the driver's decision to

$$
F_{2}=\left\{\begin{array}{l}
1 \quad P_{i} \geq P_{M A X} \\
\frac{P_{M A X}-P_{i}}{P_{M A X}-P_{M I N}} \\
0 \quad P_{M I N} \geq P
\end{array} \quad P_{M I N} \leq P_{i} \leq P_{M A X}\right.
$$

Perform a first-level comprehensive evaluation of each factor, and substitute the index data $p$ into the membership function of the two decisions according to the positive and negative of

$$
\left(R_{i j k}\right)_{n \times m} \quad(i=1,2, \cdots, n ; j=1,2, \cdots, 7 ; k=1,2)
$$

The second-level fuzzy evaluation mainly reflects the importance of the relative influence between the factors. By multiplying the weight matrix obtained from problem one and the result of the first-level fuzzy evaluation, the same

$$
B_{i k}=A_{j} \cdot R_{i j k} \quad(k=1,2)
$$

In fuzzy comprehensive evaluation, the principle of maximum membership is used to compare the membership of two decisions in the same situation. Choose the decision plan where the larger data is located. Let $B_{i 1}$ denote the degree of factors are determined as follows:

$\mathrm{P}($ Urban impact $)=$ (Impact of online car hire,

Indoor congestion )

$\mathrm{P}($ Impact of the airport $)=($ Flight arrivals, Number of vehicles in the storage pool)

$\mathrm{P}($ Temporal Climate Impact $)=($ Time, Weather impact, Solar terms effect)

Due to the strong subjective factor of membership function. And the results obtained by different functions are not the same, so the general extreme membership function is used. The membership function of the positive and negative indicators for the driver's stop-and-wait decision is as follows:

$F_{1}=\left\{\begin{array}{l}1 \quad P_{i} \geq P_{M A X} \\ \frac{P_{M A X}-P_{i}}{P_{M A X}-P_{M I N}} \\ 0 \quad P_{M I N} \geq P\end{array} \quad P_{M I N} \leq P_{i} \leq P_{M A X}\right.$

leave the airport is as follows:

$$
F_{2}=\left\{\begin{array}{l}
1 \quad P_{i} \geq P_{M A X} \\
\frac{P_{i}}{P_{M A X}} \quad P_{M I N} \leq P_{i} \leq P_{M A X} \\
0 \quad P_{M I N} \geq P
\end{array}\right.
$$

the index. Let ${ }^{R_{i j k}}$ represent the membership of index $i$ to decision $j$ in case $k$.According to this, the membership matrix expressions of each indicator for the two decisions are as follows:

situation can be obtained for two kinds of decisions. Let $B_{i k}$ denote the membership of case $i$ in the case of decision $k$, and let $A_{j}$ denote the weight of decision index $j$.Can get: :

membership of case $i$ to the no-load return decision, and let $B_{i 2}$ denote the degree of membership of case $i$ to the decision to wait for a passenger. Can get:

$$
\text { Driver selection strategy }\left\{\begin{array}{l}
\text { No-load return } \quad\left(B_{i 1}<B_{i 2}\right) \\
\text { Either case }\left(B_{i 1}=B_{i 2}\right) \\
\text { Waiting for passengers }\left(B_{i 1}>B_{i 2}\right)
\end{array}\right.
$$


5. Case Study Based on Shanghai Pudong Airport

We combined the design of 16 kinds of index data in the fourth part of the article, taking taxi drivers in Shanghai Pudong Airport as an example, collecting relevant data, applying fuzzy evaluation decision model, and quantitatively discussing the decision-making schemes of Shanghai Pudong Airport taxi drivers, and analyzed the rationality of the model.

\subsection{Data analysis}

5.1.1 Time distribution characteristics of flights at Pudong Airport
According to the intent, the taxi driver can observe the number of flights arriving within a certain period of time. The landing time of each flight and the number of passengers landing on the flight are conducive to the driver's decision. Therefore, from the Shanghai Airport official website, the number of flights arriving and departing from Pudong Airport during the three days from September 12 to 14,2019 was collected. The proportion of the number of flights arriving or departing at each time period in the day of the day is calculated in the line chart, as shown in Fig.4.

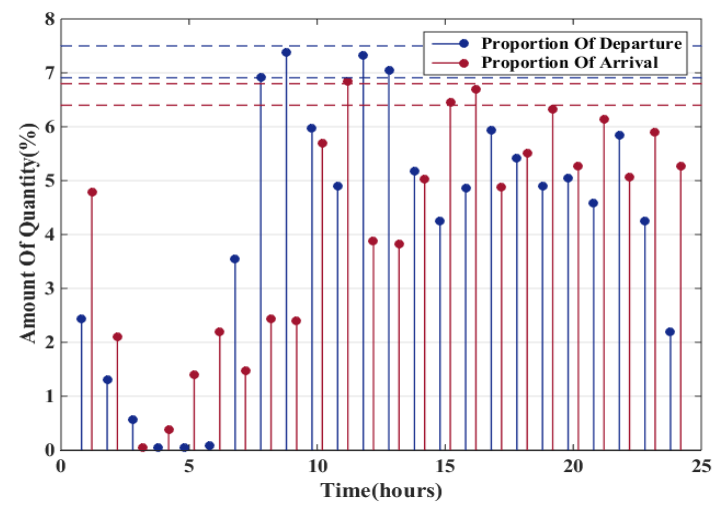

Fig 4 Stem map of arrival and departure flights at Pudong Airport in each period

Focusing on the number of flights arriving at the airport and selecting the peak value of departure flights, from the perspective of taxi drivers who are preparing to make decisions at the airport, they are more concerned about the peak period of landing flights. Observe that the time period with the largest red-blue drop is between 12:00 and 14:00. At this time, there are the most passengers leaving the airport and the least passengers arriving at the airport. At this time, the driver may consider waiting in the waiting area.

\subsubsection{Analysis of spatial clustering character-} istics of airport taxis

The following will reflect the GPS data of multiple groups of taxis near Pudong Airport at 16:00 in the coordinate system to analyze the distribution of taxi density around Pudong Airport. It could be seen from the information that Shanghai Pudong Airport is located at 31 degrees 09 minutes north latitude and 121 degrees 47 minutes east longitude. It analyzes the distribution around the airport during the peak period with graphics.

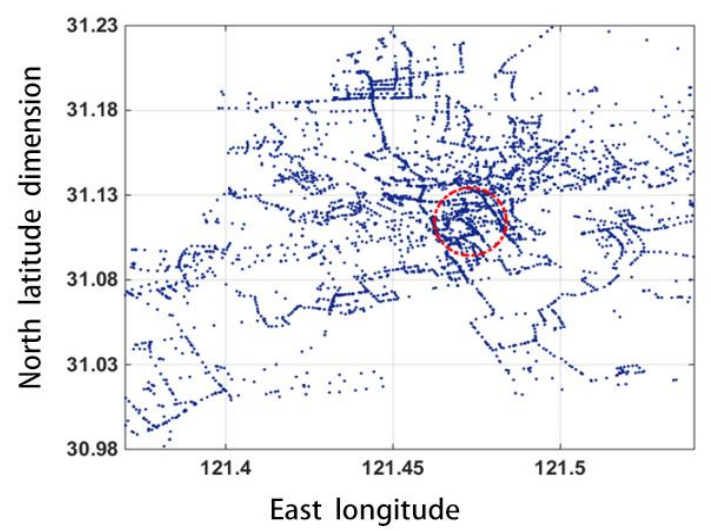

Fig 5 Distribution map of taxi locations during peak hours 
The general practical significance of the picture above is: At the time of flight arrival and departure, the public's demand for distribution is high, and the spatial distribution of taxis in this sense is in high agreement with the demand. It shows that taxis have become one of the important components of roadside public transportation in the hub airport.

\section{ons in Shanghai}

Further use K-Means ${ }^{[17]}$ to explore the distribution of taxi locations in the urban area of Shanghai, so as to understand the characteristics of taxis in Shanghai. The following Fig. is the location distribution map of all taxis in Shanghai at 16:00 on February 20, 2019 using cluster analysis.

\subsubsection{K-means cluster analysis of taxi locati-}

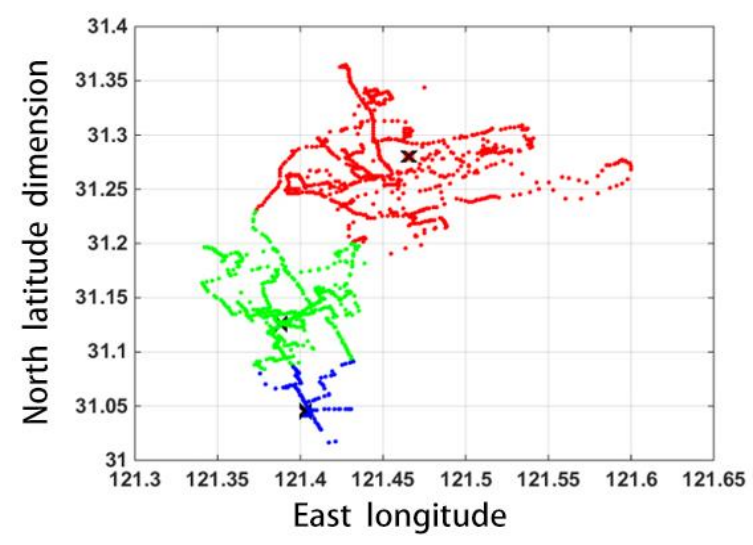

Fig 6 Taxi location distribution clustering results

Combining the actual situation and clustering results, the following conclusions were obtained:

(1) The red scatter cluster center is Shanghai Bund, and there are many attractions with attractive attractions distributed around it. The crowd of people is high and the demand for traffic is correspondingly increased. Therefore, taxis have a higher density distribution in this space.

(2) The green scatter cluster center is a commercial core area. While meeting the shopping needs of urban residents, it has also formed a high-density concentrated area. Taxi transportation methods play an important role in meeting the travel needs of such people.

(3) The blue scattered dots represent the gathering area of the science and technology park, which is the core area of Shanghai's hightech industrial zone. Other modes of transportation in this area have a higher alternative to taxis, so compared with other taxi density centers, the concentration density of technology parks is low.

\subsection{Data analysis-based instance setup}

Based on the data of Shanghai Pudong Airport, due to the complex decision-making situation faced by taxi drivers, several typical actual situations were selected for quantitative analysis based on data analysis ${ }^{[15]}$. Set the size of different indicators for the main factors affecting the decision., See the table below for specific data:

Table 2 Typical example decision index size

\begin{tabular}{c|c|c}
\hline Setting indicators & Typical data & Unit \\
\hline Size of storage pools & 300,600 & Each \\
Number of flights arriving at the airport & 2,6 & $\%$ \\
Impact factors for online car impact system & $0.25,0.75$ & $\backslash$ \\
Impact factors for seasons and weather & $0.2,0.6$ & $\backslash$ \\
\hline
\end{tabular}

The combination of pairwise indicator data can get $2^{*} 2^{*} 2 * 2=16$ typical reference situations. In 
summary, a data matrix is constructed to represent the prerequisites for airport decision-making under each combination, and the matrix $P_{i j}$ represents the value of the j-th index in the $\mathrm{i}$-th case.

\subsection{Model solving}

By giving different data sizes to different decision indicators, 16 types of indicator data are combined, and the actual membership de-retention membership is calculated using MATLAB.

Table 3 Part of the actual situation of the degree of membership

\begin{tabular}{ccc}
\hline & Membership to departure airport & Membership in leaving decisions \\
\hline 1 & 0.4564 & 0.3971 \\
2 & 0.5024 & 0.3511 \\
3 & 0.4839 & 0.3696 \\
4 & 0.5299 & 0.3236 \\
5 & 0.1735 & 0.6779 \\
6 & 0.2195 & 0.6319 \\
7 & 0.201 & 0.6504 \\
8 & 0.247 & 0.6044 \\
9 & 0.5331 & 0.3204 \\
10 & 0.5791 & 0.2744 \\
11 & 0.5606 & 0.2929 \\
12 & 0.6066 & 0.2469 \\
13 & 0.2501 & 0.6013 \\
14 & 0.2961 & 0.5553 \\
15 & 0.2776 & 0.5738 \\
\hline
\end{tabular}

According to the degree of membership in the detention plan should be selected. The table beabove table, the taxi driver can determine which low shows the results of some detention plans.

Table 4 Driver decisions in different situations

\begin{tabular}{cccc}
\hline Actual situation type & Final decision & Actual situation type & Final decision \\
\hline 1 & No-load return & 9 & No-load return \\
2 & No-load return & 10 & No-load return \\
3 & No-load return & 11 & No-load return \\
4 & No-load return & 12 & No-load return \\
5 & Stay waiting & 13 & Stay waiting \\
6 & Stay waiting & 14 & Stay waiting \\
7 & Stay waiting & 15 & Stay waiting \\
8 & Stay waiting & 16 & Stay waiting \\
\hline
\end{tabular}

\subsection{Bayesian model-based rationality test}

The driver's decision is divided into stop and wait or leave without load. This decision is regarded as a 0-1 classification problem. Bayesian theory is introduced [18]. Combining the characteristic attribute values of relevant factors that influence decision making, a naive Bayes algorithm classifier is designed to make decisions based on the relevant factors.

Let $x=\left\{a_{1}, a_{2}, \mathrm{~L}, a_{m}\right\}$ be an item to be classified, Where each $a$ is a characteristic attribute of $x$.Category collection $C=\left\{y_{1}, y_{2}, \mathrm{~L}, y_{n}\right\}$. According to Bayes' theorem:

$$
P(B \mid A)=\frac{P(A \mid B) P(B)}{P(A)}
$$


Calculate the conditional probability of the deci- leave without load:

sion label, that is, whether to choose to wait or

$$
P\left(y_{1} \mid x\right), P\left(y_{2} \mid x\right), \mathrm{L}, P\left(y_{n} \mid x\right)
$$

The result of the naive Bayesian classification satisfies the following formula decision is a decision classification label that

$$
P\left(y_{k} \mid x\right)=\max \left\{P\left(y_{1} \mid x\right), P\left(y_{2} \mid x\right), \mathrm{L} P\left(y_{n} \mid x\right)\right\}, \quad x \in y_{k}
$$

The first fourteen sets of data from the results of the secondary fuzzy discriminant model are used as the original training data for naive Bayes discrimination. Using the last two groups as test data, the driver's behavior of leaving the station is recorded as 0 , and the behavior of waiting and waiting is recorded as 1.Using MATLAB to design a Bayesian classifier, the expression value corresponding to each influencing factor is the characteristic attribute of each case, 0,1 is the set of categories. The formulas discussed in the above classification methods can be calculated by the program. After calculation, the classification results of the last two types of test data are 1,1 , and the corresponding classification results are stay-wait, which is consistent with the results of the second-level fuzzy discrimination model, which illustrates the rationality of the model.

\section{Evaluation of the model}

\subsection{Model advantages}

The full use of R-Means cluster analysis, KMeans and other cluster analysis has greater scalability and efficiency for data preprocessing, which is conducive to strengthening the effectiveness of data analysis.

The selected indicators of taxi drivers are more representative, and the indicators with less influence are excluded, so that the decision-making psychology of airport taxi drivers can be summarized more accurately.

The model and conclusions in this paper are not only applicable to the decision-making problem of airport taxi stop, but also can be used to study the decision-making strategies of other wideranging hubs, such as the related traffic arrangements at train stations and passenger stations.

\subsection{Disadvantages of the model}

The use of analytic hierarchy process will carry a certain subjective will, and the possible results obtained will be different from the actual decision-making results of taxis at sea airports.
Because the research is Shanghai Pudong Airport, only one case is selected for discussion, and it does not cover the research of different regions and airports of different sizes.

\section{Conclusion}

This article first explores the impact mechanism of taxi driver decisions and determines the relevant impact indicators that affect the decision of taxi drivers arriving at the airport. After screening some indicators, the analytic hierarchy process is used to obtain the weight of the relevant indicators. After comparing 16 empirical examples in Shanghai, the fuzzy decision is used to discuss the decision-making strategies of airport taxi drivers. The most important factors that can determine whether the taxi driver stays at the airport are the number of arriving flights, the number of vehicles in the storage pool, and the effect of morning and evening time. The factors that have a greater impact on airport taxi drivers are the impact of the weather on the day, the congestion in the city, and the impact of online car hire. The factors that have less impact on airport taxi drivers are the seasonal impact of the year, the impact of major events, and the impact of automobile oil prices. If there are a large number of vehicles in the car storage pool, the proportion of flights is small, due to the large number of competitive vehicles at the airport. Taxi driver will choose to return to the city to solicit guests. As the number of flight arrivals has a great influence on drivers' decision-making, and the impact of online ride-hailing is relatively small, the main criterion of drivers' decision-making is still waiting when there are a large number of flights at the airport, that is, when the proportion of flights is large.

\section{References}

1. Ting Wu, Research on Optimal Cruise Path Modeling Based on Taxi Cruise Mechanism Analysis, 
2019, Jilin University.

2. Chaogui Kang, et al. Impact of weather factors on taxi travel in Wuhan. Journal of Geo-Information Science, 2019. 21 (01): 118-127.

3. Dimitriou, L., et al., Dynamic Estimation of Optimal Dispatching Locations for Taxi Services in Mega-Cities based on Detailed GPS Information. IFAC PapersOnLine, 2016. 49 (3).

4. Maguire, E.A., K. Woollett and H.J. Spiers, London Taxi Drivers and Bus Drivers: A Structural MRI and Neuropsychological Analysis. Hippocampus, 2006. 16 (12): p. 1091-1101.

5. Bao, Feng Study on the Method for Determining the Scale of Taxi Pick-up and Drop-off Points in Large Comprehensive Transportation Hubs. Urban Roads, Bridges and Flood Control, 2019 (07): $45-48+52+9-10$

6. Yong Han et al. Study on the Spatiotemporal Distribution Characteristics of Rental Passenger Points Based on Clustering Algorithm. Journal of Ocean University of China (Natural Science Edition), 2019. 49 (S1): 155-162.

7. Sirui Lin, Research on Forecasting Demands of Airport Taxi Capacity, 2018, University of Electronic Science and Technology of China.

8. Bingjie Wang, Kezhen Wang, and Gang Han, Study on Airport Taxi Driver Decision Model Based on AHP. Journal of Baicheng Teachers College, 2019. 33 (10): 23-25 + 29.

9. Jing, Y., et al., Understanding Collective Human Mobility Spatiotemporal Patterns on Weekdays from Taxi Origin-Destination Point Data. Sensors (Basel, Switzerland), 2019. 19 (12).

10. XU, M., et al., Mobility Pattern of Taxi Passengers at Intra-Urban Scale: Empirical Study of Three Cities. Journal of Systems Science and Information, 2017.5 (06): p. 537-555.

11. Maguire, E.A., K. Woollett and H.J. Spiers, London Taxi Drivers and Bus Drivers: A Structural MRI and Neuropsychological Analysis. Hippocampus, 2006. 16 (12): p. 1091-1101. characteristics of passenger OD in Beijing's foreign transportation hub. Journal of Geoinformatics, 2016.18 (10): 1374-1383.

13. Tong Wang and Zhaoyi Shen, Research on Hotspot Technology for Taxi Passengers. Journal of Harbin Commercial University (Natural Science Edition), 2019. 35 (05): 590-594 + 606.

14. Machin, M.A. and J.D. Souza, Predicting health outcomes and safety behaviour in taxi drivers. 2004. 7 (4): p. 257-270.

15. Chao Yan, Study on Landside Public Transportation Management of Shanghai Hub Airport, 2015, East China Normal University.

16. Jun Ye, Qiyuan Jiang, Jinxing Xie, Mathematical Models, 4th Edition Electronic Lesson Plan. 2011: Beijing: Higher Education Press.

17. Zhiguo Zhao et al. Improved K-means clustering and recognition of driver's collision avoidance steering behavior. Automotive Engineering, 2020 (01): 52-58.

18. Yin Guan, Xinyue Li and Jiaming Zhu, Bayesian Discriminant Analysis of Manufacturing Financial Early Warning. Journal of Neijiang Normal University, 2019. 34 (06): 68-72.

2. Yang Gege, et al. Spatiotemporal distribution 